\title{
The System Design of Full Servo Planetary Pole Symmetric Pipe Cutting Machine
}

\author{
Wan Xu, Cong Li, Di Zhao, Jiong Li, Yingping Qian \\ School of mechanical engineering, Hubei University of Technology, Wuhan, 430068, China
}

Keywords: pole symmetric; planets operation, servo drive straight, PLC control system, automatic cutting.

\begin{abstract}
According to the cutting principle of the planetary pole symmetric structure, in the paper, it makes the planet cutting as the main movement, to control the cutting chips and the smoothness of the cutting section. The system contains six axes, it adopts full servo direct driving, and uses electronic gear to control the pulse equivalent to ensure accuracy. The system is controlled by PLC, including the automatic, the manual and the semi-automatic, which can satisfy the demand of industrial production. Through the touch screen communication, the operation has become more simple. The system has good reliability, simple circuit, flexible control, and strong practicability.
\end{abstract}

\section{Introduction}

At present, the application of stainless steel is more and more widely in China. As special steel, it is resistant to high temperature fatigue and acid corrosion. It is one of the preferred car exhaust pipe material. On the premise of meet the technological requirements, cutting the long steel for the fixed-length steel materials has become a primary part in the steel pipe manufacturing.

At present, Cutting methods in China mainly include grinding wheel cutting, laser cutting, wire cutting, single pole coil type cutting, etc. Of these, the grinding wheel cutting currently applied in the general steel pipe cutting is more, but it is difficult to realize high precision and high efficiency; Although laser cutting [1] can achieve high accuracy, high efficiency, but because of the high price of the power laser head, high temperature produced with a little danger when cutting, it is not suitable for general industrial production. The wire cutting can achieve high precision, but with low cutting efficiency; For the cutting method of the single blade with pipe rotating [2], because the pipe itself is rotating, it is difficult to ensure the precision when cutting. The cutting method the paper mentioned not only can realize high speed and high precision cutting with well cutting chips, but also the price is cheap, applicable to the general industrial production line.

PLC as controller of specially designed for industrial environment, it is tightly solid structure, small volume, light weight, low power consumption, and anti-jamming capability is strong. And it is mainly applied to discrete manufacturing and process manufacturing, etc. [3]. PLC and servo direct driving is used in the system [4], the way of working has simple structure, accurate positioning, and it is easy to control. It can ensure the reliability of the work and achieve high cutting accuracy.

\section{The principle of the cutting machine and the selection of the servo}

\subsection{Structure analysis.}

The mechanical structure of this system is mainly composed of feeding and cutting.

Feeding section contains a feeding screw and a feeding clip, cutting section contains a front clip and a cutter-head to finish the cutting. The clips adopt automatic central type grip design, to ensure the concentric of the clips during the automatic clamping.

Cutting section adopts planetary pole symmetric design [5-6]. That is rotating itself $\longrightarrow$ linear motion $\rightarrow$ revolution. The main idea is that the blades rotate first, then the feeding motors complete the straight feeding, and at last, the blades revolute a half round with the cutter-head rotating to finish the cutting. Calibrating two blades on the same plane before the cutting can get the 
cutting face with high quality. And because the two blades are cutting at the same time, to a certain extent, compared with the single blade, it improves the cutting efficiency.

The overall station diagram of the pipe cutting machine is shown in Fig.1.

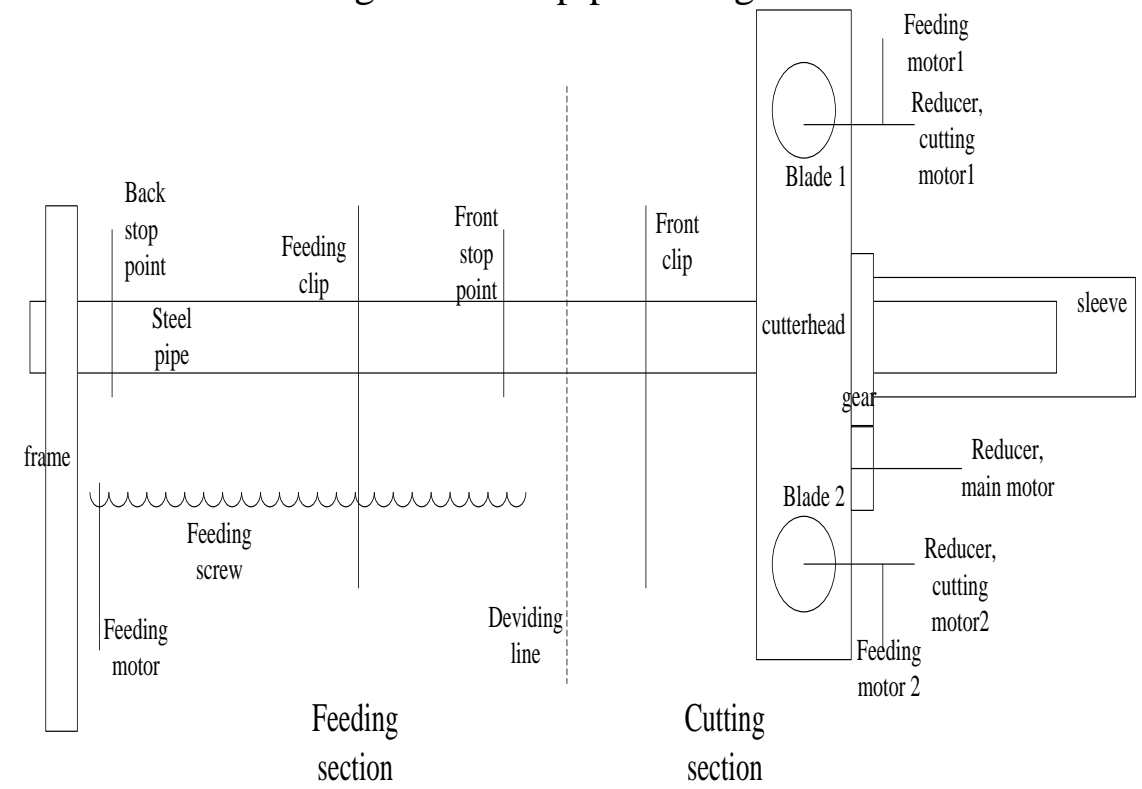

Fig.1: The overall station diagram of the pipe cutting machine

\subsection{The working process.}

The working process of the machine mainly includes the following four steps:

i) Clamp the feeding clip. Putting the pipe on the frame and the feeding clip, then the feeding clip completes the clamping.

ii) Feeding and then clamp the front clip. The feeding clip sends the pipe to the fixed position (the actual length of the required cutting + the thickness of the blade), and then the front clip clamps.

iii) The blades rotate and then complete feeding. Blades 1 and 2 begin to rotate with clockwise direction (from the feeding direction). Meanwhile, the feeding motors 1 and 2 send the blades to the surface of the pipe, the paper defines the distance as fast feeding distance. (in the automatic mode, adjust the distance manually, and set it on the screen). And then slow down to the work speed to work some distance, the distance (called working distance) is determined by the thickness of the pipe.

iv) Complete the cutting and then the blades retract. After the work above, the cutter-head rotates with counter-clockwise direction ( from the feeding direction). Because the cutter-head has two symmetrical blades, so it generally needs the cutter-head to rotate $170^{\circ}$ to complete a cutting.

Theoretically, the cutter-head can be changed to a clockwise rotation (from the feeding direction ) as the second cutting, and by changing the rotating direction of the blades to cutting burr incoming or exterior pipe. However, due to the assembly fixed of the blades, the direction of the rotation of the blades is fixed. So at the step of program designing, before the second cutting, the blades and the cutter-head must return the starting point and stop rotating.

In order to maintain the continuity of the movement and reduce the wear of feeding screw, this system makes full use of the feed screw, the back stop point is the feeding starting point, front stop point is the feeding end point. After completing the first cutting, loosening the front clip, not loose feeding clip, and then continue to the second cutting.

\subsection{The selection of the drive servo system.}

According to Fig.1, the system requires six sets of servo driving system. The feeding and the rotation of cutter-head can be realized by the position control of the servo motor, and the cutting module adopts the analog control to change the torque and rotational speed. The system selects 
Mitsubishi's servo amplifiers and corresponding servo motors. The models selected is shown in Table 1.

Table 1: The selected models

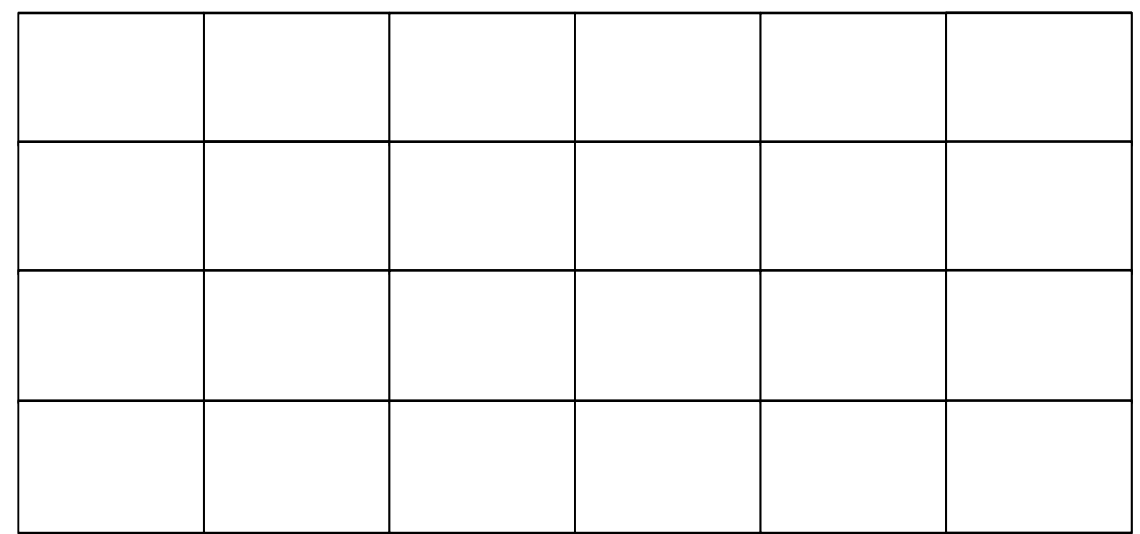

\section{Control system design}

\subsection{Control requirements.}

In this paper, the main function to achieve includes:

i) Manual and automatic functions: not only it can jog control each step, to achieve initial debugging capabilities, but also can achieve one-button control, to increase productivity.

ii) The stable control of each movement trajectory, which is the key of the motion control.

iii) Facilitate human-machine communication: fool-button operation can help workers control the equipment easily.

\subsection{PLC selection and $\mathrm{I} / \mathrm{O}$ assignment.}

The system selects the FX3U series PLC produced by Mitsubishi. The method of PLC control is pulse + direction. According to section 2.3, the system requires four pulse output, but the PLC only has three high-speed pulse output ports, so it needs for a additional pulse output channel. The paper has selected FX2N-1PG for a pulse generator to control the movement of the cutter-head.

Because the torque and rotational speed are control by analog, so it need four analog output port on the control module. The paper has selected FX2N-4DA for the analog output module. The overall I/O allocation of the system and the overall scheme are shown in Fig.2.

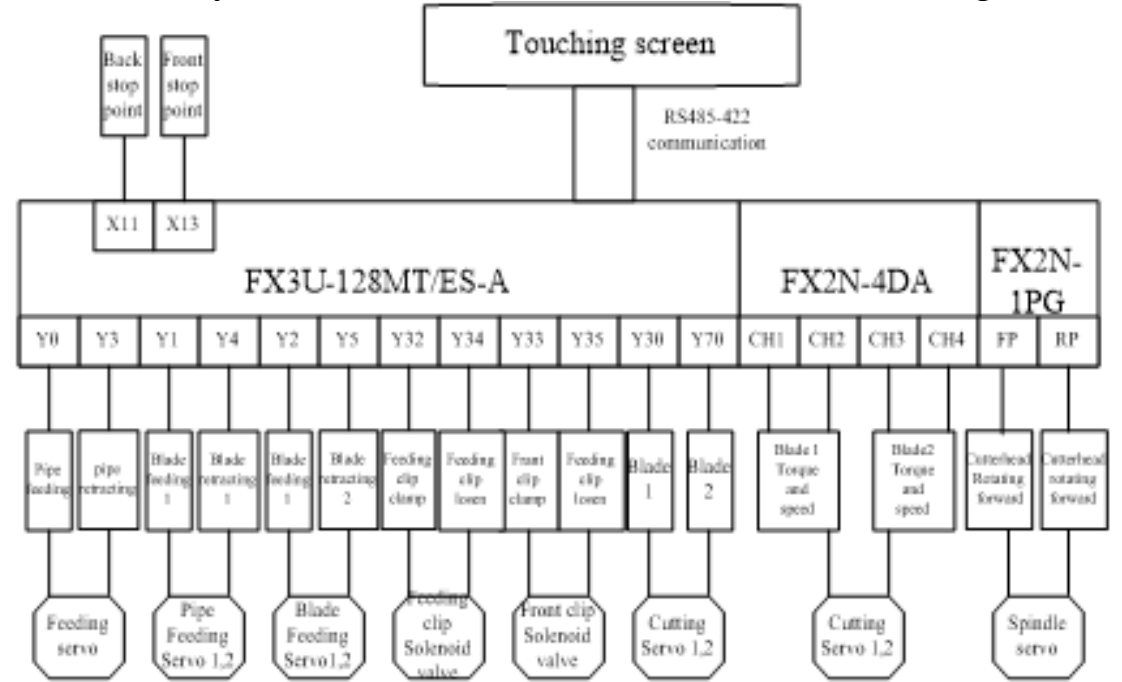

Fig.2: The overall I/O allocation of the system and the overall scheme 
In addition, due to the cutting process will produce a lot of heat, it is also need a cooling device to cool the blades. Considering the operation convenience, the cooling equipment is controlled with manual push-button.

\subsection{Control program design}

Control program design method is sequential function chart. The design idea based on SFC is to divide a duty cycle of the system into a number of stages connected in order. The design approach allows a complex production process into several steps, it not only simplifies the programming of the clarity, enhances readability, but also helps the developers to debug, modify, and the maintenance of equipment.

Using the software of GX-Developer and GX-Simulink, it can do program simulation and debug out of PLC to a certain extent. Through such a development platform, the internal parameters of PLC, motion programs and the motion state can be monitored in real time.

The program section of the cutting machine consists of the initial program and the main program. The initial program is used to set boot defaults and modify the parameters of the touch screen, etc. The main program flow of the system is shown in Fig.3.

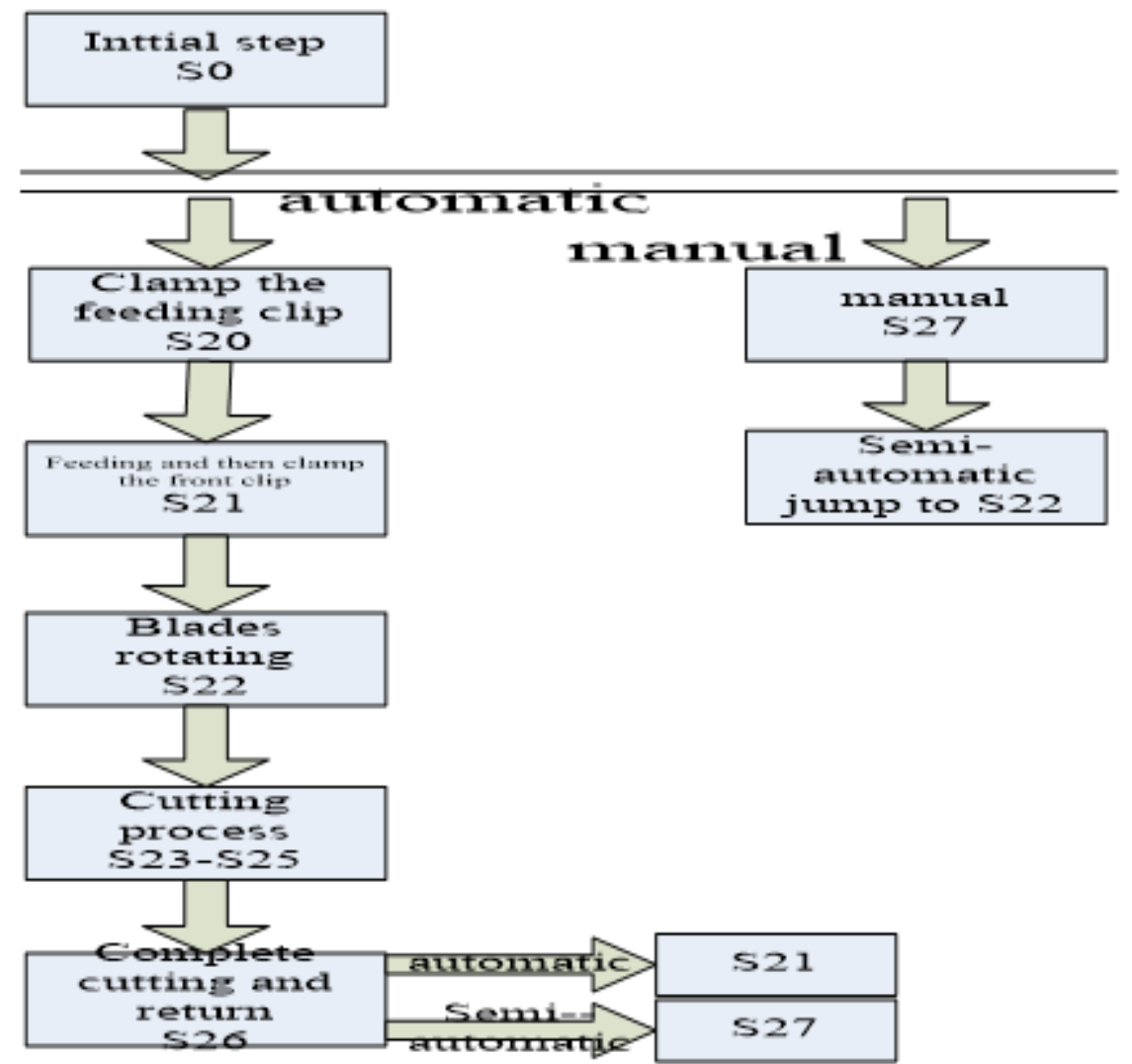

Fig.3: The main program flow of the system

There are three kinds of control modes, including manual, semi-automatic control and automatic control. The manual is mainly used for initializing and equipment debugging after the boot; the semi-automatic is mainly used for the first cutting before the automated production; the automatic is used for automated production process.

\subsection{Servo drive debugging}

Throughout the system, the setting of the servo parameter has great influence on the working state and the motion accuracy. Adjustment mainly includes three aspects:

i) Control mode selection:

For the pipe feeding, blade feeding and the cutter-head motion, the control mode is selected for the position control, and for the rotational motion of the blade, the control mode is torque control. 
ii) Electronic gear selection:

The electronic gear ratio $(\mathrm{CMX} / \mathrm{CDV})=$ the detecting feedback pulses of the encoder/the commanding pulses from PC. When to have certain control precision requirement, it can determine the ratio according to the pulse equivalent and the detecting feedback pulses of the encoder, to achieve high precision. The paper has selected a screw which the lead is $10 \mathrm{~mm}$. By adjusting the electronic gear ratio, setting the command pulse equivalent to $0.001 \mathrm{~mm}$, in the case of the servo without steps losing, the highest precision can reach $1 \mathrm{um}$, meeting the production requirement.

iii) The servo's self-learning

The self-learning is generally used in the changed from light to load, because it may be abnormal in this time, so the self-learning can be able to learn by adaptive PID to solve.

\section{Human-machine interface design}

With the improvement of the automation degree of modern equipment, human-machine interface plays a more and more important role.[7]

The system adopts the touch screen of the series of TK, WEINVIEW, the software it chosen to design is EB8000 Project Manager, and modular programming method is used. By giving module the corresponding address and data, developers can download the program to the touch screen after finished the offline and online simulation. In the offline simulation, PLC can not be connected, but it needs to connect PLC in the online simulation. The program designing flow is shown in Fig.4.

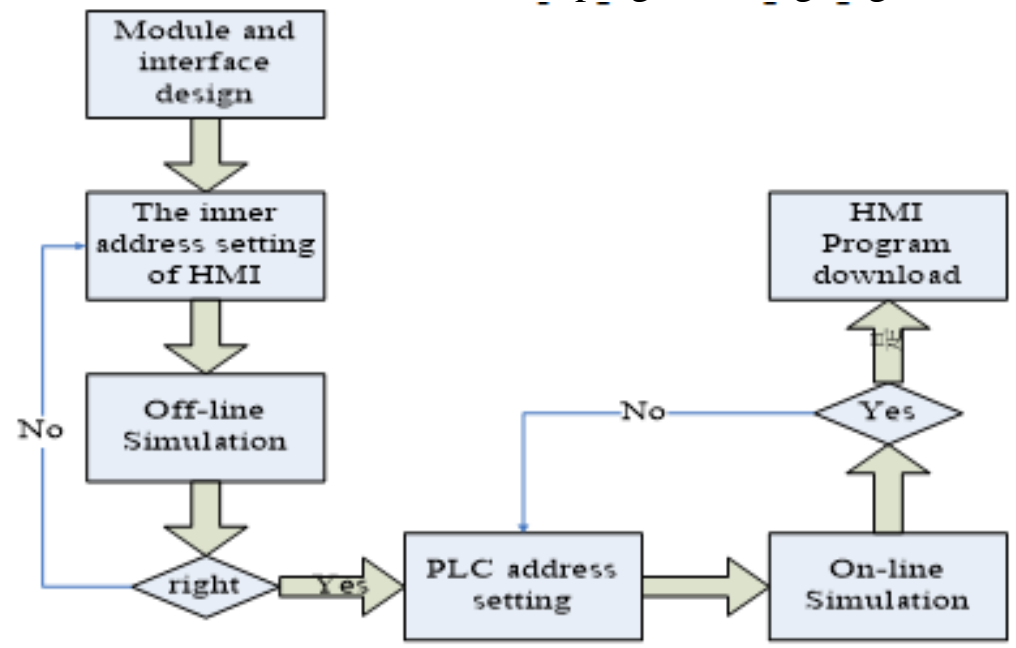

Fig.4: The program designing flow.

In the system, communication between man-machine interface and PLC via industrial PC serial port (RS485/RS422) and the PLC communication module (FX3U-422-BD). The system can easily be controlled by human-machine interface and data modification, debugging.

\section{Conclusion}

The pipe cutting machine in the paper adopts the hydraulic clamping control and full servo driving control. It is effective to achieve a desired action, and can get a certain control precision, which's cutting accuracy can reach to 1 um. In addition, it can also get a cutting section with high quality. The equipment to some extent, make up for the shortcomings of traditional cutting accuracy, improve the cutting section quality and cutting efficiency. With the tungsten steel blade, the time of completing a cutting has decreased to 2 minutes from $10 \mathrm{~min}$, the fever is small, and the capacity has got a big promotion. The equipment has been designed and put into use.

\section{Acknowledgements}

The research work was supported by National Natural Science Foundation of China under Grant No. 51405144 and Natural Science Foundation of Hubei Provincial under Grant No. 2014CFB598. 


\section{References}

[1] Jijiang Xie, The design of the industrial CNC laser cutting machine. Optical and Precision Engineering, 12(6),pp.587-591,2004.

[2] Shulan Ling, The design and pratice of the cutting machine with the piep rotating. Coal Mine Machinery, 30(2),pp.30-32,2009.

[3]Wenjie Zhu, Mitsubishi FX Series PLC Programming and Application. Beijing: China Electric Power Press. 2013.

[4] Shengdun Zhao, The rearch of the AC servo driect driving of the turret press mechine. China Metal Forming Equipment \& Manufacturing Technology, (6), pp.19-22,2013.

[5] Jun Liu, The rearch of the plantary CNC chipless cutting machine. Shenyang University of Technology. 2012.

[6] Xu huang, The rearch of the thin-walled stainless steel pipe cutting methods and equipment. Hubei University of Technology. 2014.

[7] Yanmei Liu, The Base of The Mitsubishi PLC and System Design (The Second Edition). Beijing: Mechanical Industry Press. 2012. 